\title{
Improvement in electro-optical and dielectric characteristics of ZnO nanoparticles dispersed in a nematic liquid crystal mixture
}

\author{
AMIT SHARMA ${ }^{1,2}$, PRAVEEN MALIK ${ }^{1, *}$, RAVINDRA DHAR $^{3}$ and PANKAJ KUMAR ${ }^{4}$ \\ ${ }^{1}$ Liquid Crystal Research Laboratory, Department of Physics, Dr B R Ambedkar National Institute of Technology, \\ Jalandhar 144011, India \\ ${ }^{2}$ Arni School of Technology, Arni University, Indora 176401, India \\ ${ }^{3}$ Soft Material Research Laboratory, Centre of Material Sciences, University of Allahabad, Allahabad 211002, India \\ ${ }^{4}$ Department of Applied Sciences, Chitkara University Institute of Engineering and Technology, Chitkara University, \\ Jhansla, Rajpura, Patiala 140401, India \\ *Author for correspondence (malikp@nitj.ac.in)
}

MS received 30 August 2018; accepted 29 March 2019

\begin{abstract}
Reported herein is the effect of dispersion of a fixed amount of 0.5\% (wt/wt) ZnO nanoparticles (NPs) in a nematic (E7) liquid crystal (LC) mixture. Dispersion of ZnO NPs results in a significant improvement in electro-optical and dielectric parameters of the nematic mixture. Comparative analysis of undoped and NP-dispersed samples shows a reduced threshold voltage with better transmittance without compromising dielectric permittivity characteristics. In addition, an increase in the contrast ratio, birefringence, alternating-current conductivity and band gap was observed after dispersion of ZnO NPs in LCs. A polarized optical microscopic study of the NP-dispersed sample substantiates a slight increase in nematic-isotropic phase transition temperature of LCs.
\end{abstract}

Keywords. Nematic liquid crystals; $\mathrm{ZnO}$ nanoparticles; dielectric permittivity; threshold voltage; conductivity.

\section{Introduction}

Contemporary research on liquid crystal (LC) materials is continuing over the last few decades due to their wide range of applications. LCs, one of the organic materials having interesting electro-optic properties, play a key role in the advancement of current display technology like counting wrist watch to three-dimensional display devices [1-8]. LCs have also been used in electro-optic filters, holography, digital data storage and biosensors $[9,10]$. Thus a wide use of LC displays arises a demand for the investigation of new LCs which led towards more perfection in display devices. In the bargain of new LCs, dispersion of the guest material in LCs is a supportive technique to obtain the desired properties for display applications [11]. Consequently, a LC-nanoparticle (NP) composite is one of the most desired approaches to improve physical and electro-optical properties of LC devices. As dispersion of NP in LCs is cost-effective, and enables to produce new LC-NP composite materials having unique and improved properties that are different from pure LCs [12-20]. Thus, different types of NPs such as semiconducting, ferroelectric, metallic, inorganic, carbon-related and ferronematics are being used to produce technologically improved electrooptic devices [21-25]. The commonly used NPs with nematic LCs are $\mathrm{ZnO}, \mathrm{TiO}_{2}$, diamond, $\mathrm{BaTiO}_{3}$ and $\mathrm{Ag}$ [9,12,26-29].

However, to control a long range orientation order of LCs in the LC-NP composite material for obtaining a superior device performance is critical [3,4,6-8]. Dispersion of $\mathrm{TiO}_{2}$ NPs in nematic LCs has been analysed along with the effect of UV exposure and the suppressed ionic effect with a promoted voltage holding ratio has been reported [26]. Further, dispersion of diamond NPs in nematic LCs results in slowing down of the relaxation process, which further indicates that NPs impend the rotation of LC molecules and change the ionic concentration with an increase in dielectric permittivity and conductivity [27]. The nematic $\mathrm{LC}$ and $\mathrm{BaTiO}_{3} \mathrm{NP}$ composite system result in the formation of micro-droplets of LCs with NPs. These micro-droplets align themselves along the applied electric field and improved the electro-optic characteristics of the LC device [28]. Dispersion with Ag NPs in nematic LCs brings out a decrease in a threshold voltage with enhanced conductivity and increased nematic to isotropic phase transition temperature [29].

Carbon nanotubes are another potential material of widely used NPs, which reduced the response time, driving voltage and stabilized field-induced alignment of LCs, due to similarity in their structure and shape with LCs [27]. As well, ferroelectric NPs dispersed in the LC system demonstrated improvement in the dielectric anisotropy, birefringence and clearing temperature. A reduction in the Freedericksz transition and a change in spontaneous polarization of the ferroelectric smectic LCs with the dispersion of ferroelectric NPs are also achieved [30]. In addition, an improved response time, higher contrast ratio, PL intensity, threshold voltage, 
drop in phase delay and fast switching have been reported in quantum dot dispersed LC mixtures [31]. Coming on metallic NPs, ZnO NPs find use in a wide range of technologically important areas of LCs due to simple production, large band gap energy, high exciton binding energy and excellent piezoelectric properties at room temperature. Additionally, a decrease in the threshold voltage, lower energy consumption, controlled orientation and increased phase transition temperature of $\mathrm{LCs}-\mathrm{ZnO}$ composites is reported [9,14,29,32]. It is also observed that a decrease in the threshold voltage with dispersion of ZnO NPs is resulted by suppressing undesired field screening effects due to reduced moving ion density [13]. Thus, extensively, in this paper the effect of $\mathrm{ZnO} \mathrm{NP}$ dispersion on textural, electro-optic and dielectric behaviour of nematic LCs has been investigated and analysed. Nevertheless, on the whole it must be noted that NP dispersion in the LC system does not always bring improvements in material goods and alteration in physical as well as electro-optical properties and is related to functionality of the NPs in the LC system [33].

\section{Experimental}

A room temperature nematic LC E7 (from Merck, compositions: 4-cyano-4'- $n$-pentyl-biphenyl (51\%), 4-cyano-4'- $n$ heptyl-biphenyl (25\%), 4-cyano- $4^{\prime}-n$-oxyoctyl-biphenyl $(16 \%)$ and 4-cyano-4" $-n$-pentyl-biphenyl (8\%)) with dielectric anisotropy $(\Delta \varepsilon)=+13.8$, birefringence $(\Delta n)=0.20$ and nematic-isotropic temperature $\left(T_{\mathrm{N}-\mathrm{I}}\right)=60.5^{\circ} \mathrm{C}$ as well as inorganic $\mathrm{ZnO}$ NPs (Sigma-Aldrich) with an average particle size $\sim 50 \mathrm{~nm}$ and molecular weight $=81.39 \mathrm{~g} \mathrm{~mol}^{-1}$ were used for the experimental investigations. Planner indium tin oxide (ITO) cells (Instec, USA) with a thickness of $9 \mu \mathrm{m}$ were used. For the NP-dispersed sample, the E7-ZnO suspension was prepared by adding $0.5 \%$ (wt/wt) of ZnO NPs in E7. Chloroform was also added to the mixture. Homogeneous dispersions of the ZnO NPs in E7 were obtained by stirring overnight, followed by an ultrasonic process at a frequency of $20 \mathrm{kHz}$ for $1 \mathrm{~h}$. The mixture was then subjected to heating at about $55^{\circ} \mathrm{C}$ in a vacuum oven (Harco) and cooled at room temperature for $24 \mathrm{~h}$. Two sample cells, one for pure E7 and other for E7-ZnO composites, were prepared and then filled separately with these mixtures by capillary action at their clearing temperature. After filling, both cells were sealed with araldite. Before electro-optic and dielectric measurements, to enhance accuracy of results, the sample cells were heated and cooled from the nematic (room temperature) to isotropic phase and vice versa respectively, at a rate of $1{ }^{\circ} \mathrm{C} \mathrm{min}{ }^{-1}$.

Dielectric measurements were performed by a software (Linksys) controlled impedance analyser (Wayne Kerr, Model $6500 \mathrm{~B}$ ) in the frequency range of $20 \mathrm{~Hz}-10 \mathrm{MHz}$, attached with a temperature controller cum heating/freezing stage ( -196 to $600^{\circ} \mathrm{C}$, Linkam, Model THMS600E). Sample cells were first calibrated with an impedance analyser and dielectric data of empty cell, with benzene and LCs, were recorded as a function of frequency to calculate real and imaginary parts of permittivity. Dielectric data of LCs in the nematic phase were recorded at different temperatures during the cooling cycle. Textural and phase transition behaviour was investigated using a polarising optical microscope (POM) (Nikon, ModelLV100POL) equipped with a Q-imaging camera (Q-28378). Transmission characteristics were studied using a $\mathrm{He}-\mathrm{Ne}$ laser (Melles Griot, $5 \mathrm{~mW}, 632.8 \mathrm{~nm}$ ) and photo-detector (PD02, Instec USA) attached with a function generator (Tektronix, AFG3021B). The complete set up for the electro-optic study is shown in figure 1 . The energy band gap $\left(E_{\mathrm{g}}\right)$ was measured by a UV-visible spectrometer (Carry 100, Agilent) in the wavelength range of $200-450 \mathrm{~nm}$ at room temperature.

\section{Results and discussion}

\subsection{POM study}

Optical textures were investigated in a wide nematic phase under cross polarizers. Figure 2 shows the POM textures of E7 and E7-ZnO samples during the cooling cycle. As shown in figure $2 \mathrm{a}$, the transition from the isotropic to the nematic phase begins to appear at $60.5^{\circ} \mathrm{C}$. As the temperature decreases, the liquid crystalline phase appeares at $59.6^{\circ} \mathrm{C}$. However, on further decrease in temperature a clear nematic phase at room temperature can be observed in figure $2 \mathrm{c}$. In the case of the NP-dispersed sample, the transition from the isotropic to nematic phase was found at $61.5^{\circ} \mathrm{C}$ as shown in figure $2 \mathrm{~d}$. As the temperature decreases, liquid crystalline phases can be clearly seen at $60.9^{\circ} \mathrm{C}$ and room temperature in figure $2 \mathrm{e}$ and f respectively. The studies confirm a minute increase $\left(\sim 1^{\circ} \mathrm{C}\right)$ in nematic-isotropic phase transition temperature of E7 LCs after dispersion of $\mathrm{ZnO}$ NPs. This minute increase in nematicisotropic phase transition temperature after dispersion of NPs can be attributed to a change in the molecular orientation and intermolecular interactions between LCs and NPs, which alter the order parameter that results in enhanced thermal stability, hence increase in isotropic-nematic phase transition temperature [34].

Further, the effect of the applied voltage on textural behaviour of $\mathrm{E} 7$ and $\mathrm{E} 7-\mathrm{ZnO}$ sample cells was analysed at a frequency of $1 \mathrm{kHz}$ at $35^{\circ} \mathrm{C}$. Figure 3 shows the POM textures of both sample cells under cross polarizers at a magnification of $10 \times$. As shown in figure 3, an initial bright state in the nematic phase at $0 \mathrm{~V}$ for both E7 and E7-ZnO samples was observed. With an increase in the voltage, $0-2 \mathrm{~V}$, this bright state begins to change to a dark state and approaches a much darker state. This behaviour can be related to fact that at $0 \mathrm{~V}$, initially LC molecules have a parallel orientation with a substrate and as the voltage increases molecules start to change their orientation from parallel to perpendicular (vertical) with the substrate. Due to the vertical orientation of LC molecules with the substrate, light transmission is blocked by an analyser and the dark state appears [35]. Further, the appearance 


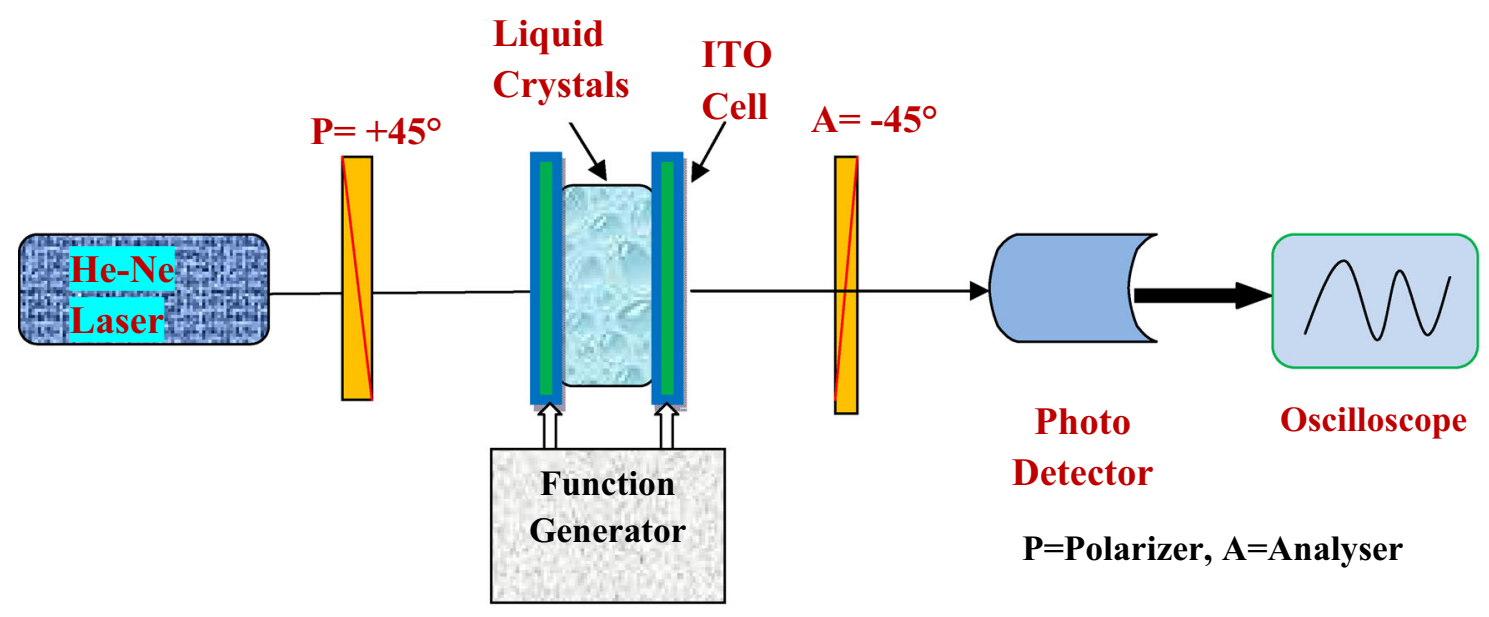

Figure 1. Experimental set-up for electro-optic measurements.

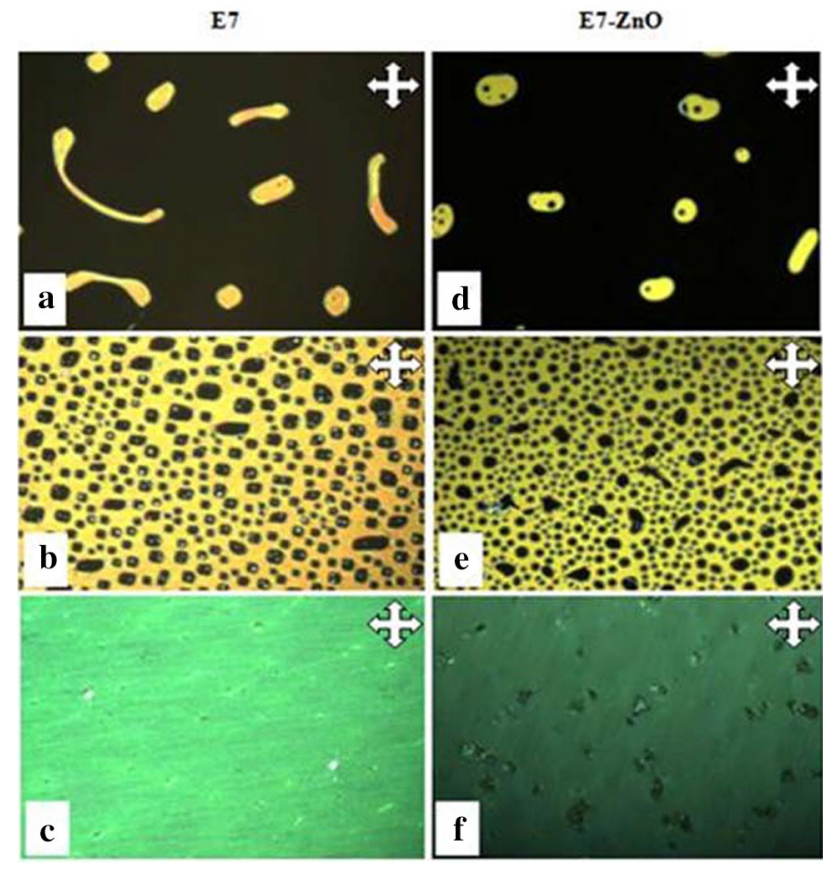

Figure 2. POM textures (a), (b) and (c) at $60.5,59.6$ and $30^{\circ} \mathrm{C}$, respectively, for the $\mathrm{E} 7$ sample. Textures $(\mathbf{d}),(\mathbf{e})$ and (f) at 61.5, 60.9 and $30^{\circ} \mathrm{C}$, respectively for the $\mathrm{E} 7-\mathrm{ZnO}$ sample. All textures were taken at a magnification of $10 \times$. Cross arrows indicate the position of the polarizer and analyser.

of dark and bright states also supported by the behaviour of voltage-transmission curves and it is discussed in the next section of electro-optic studies.

\subsection{Electro-optic studies}

Voltage-transmission $(V-T)$ characteristics of E7 and E7$\mathrm{ZnO}$ in the nematic phase at $35^{\circ} \mathrm{C}$ are shown in figures 4 and 5 .
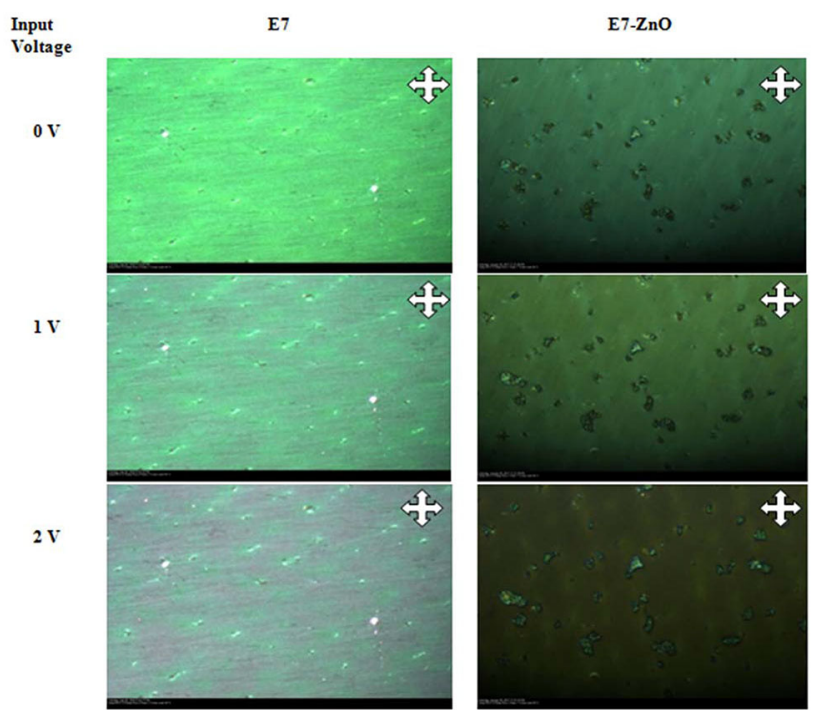

Figure 3. POM textures of $\mathrm{E} 7$ and $\mathrm{E} 7-\mathrm{ZnO}$ sample cells at $35^{\circ} \mathrm{C}$ under cross polarizers at a magnification of $10 \times$. Cross arrows indicate the position of the polarizer and analyser.

The extended view of the $V-T$ curve in a low-voltage range is also shown in the inset. From $V-T$ characteristics one can see the operating voltage of the LC cell that reflects the effect of ionic behaviour on the electro-optic properties of the LC device [26]. During the measurement of optical transmission, the LC cells were placed between crossed polarizers at an angle of $45^{\circ}$ with the optic axis of incident light. The detailed assembly of the LC cell under a polarizer, incident light and detector is shown in figure 1 . The intensity of transmitted light passing through the cell is given by $[9,35-37]$ :

$$
I_{\mathrm{t}}=\frac{\sin ^{2}(2 \theta)}{2}(1-\cos \Delta \emptyset) .
$$




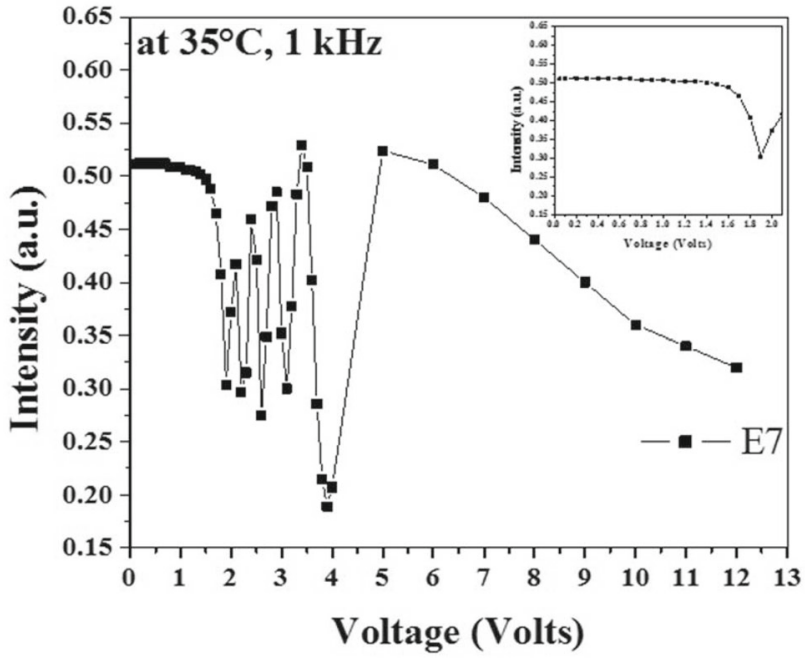

Figure 4. Voltage-transmission $(V-T)$ characteristic of the E7 sample cell under a crossed polarizer at applied voltages from 0 to $12 \mathrm{~V}$ (temperature $35^{\circ} \mathrm{C}$, frequency $1 \mathrm{kHz}$ ). The inset shows an extended view of the $V-T$ curve at a lower voltage.

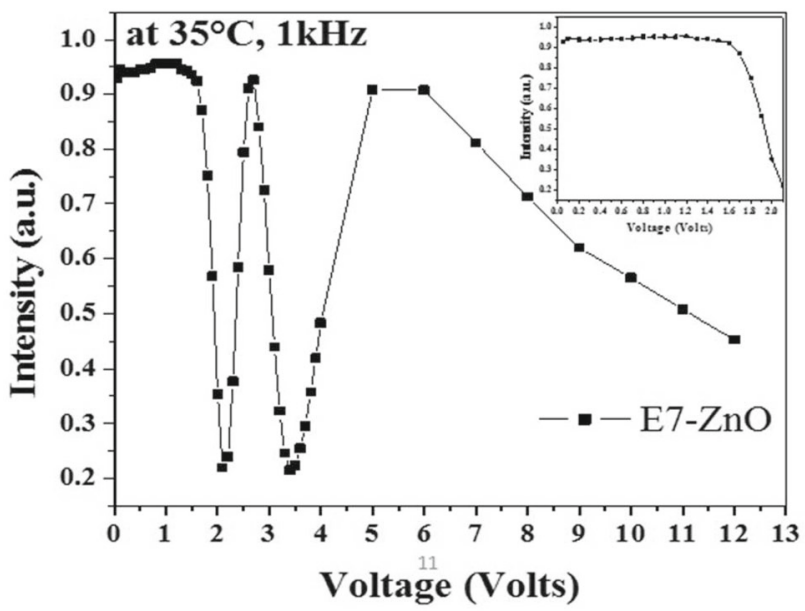

Figure 5. Voltage-transmission $(V-T)$ characteristic of the E7$\mathrm{ZnO}$ sample cell under a crossed polarizer at applied voltages from 0 to $12 \mathrm{~V}$ (temperature $35^{\circ} \mathrm{C}$, frequency $1 \mathrm{kHz}$ ). The inset shows an extended view of the $V-T$ curve at a lower voltage.

Here, $I_{\mathrm{t}}$ is the transmitted light intensity, $\theta$ is the angle between the polarizer and optical axis and $\Delta \emptyset$ is the phase difference. The birefringence $(\Delta n)$ can be calculated using $\Delta \emptyset$ from equation (1) as:

$$
\Delta \emptyset=\frac{2 \pi}{\lambda} \Delta n \cdot d
$$

Here, $d$ is the thickness of the cell and $\lambda$ is the wavelength of incident light. The angle $\theta$ is set at $45^{\circ}$ to optimize the intensity:

$$
I=I_{0} \sin ^{2}\left(\frac{\Delta \emptyset}{2}\right)
$$

$$
\begin{aligned}
\Delta \emptyset= & n \pi+2 \sin ^{-1} \sqrt{\frac{I-I_{\min }}{I_{\max }-I_{\min }}} \\
& \text { for } n=0,2,4, \ldots \\
\Delta \emptyset= & (n+1) \pi+2 \sin ^{-1} \sqrt{\frac{I-I_{\min }}{I_{\max }-I_{\min }}} \\
& \text { for } n=1,3,5, \ldots
\end{aligned}
$$

In the above equations ( $3 b$ and $3 b$ ), $n$ is the number of maximum peaks observed in the $V-T$ curve, $I$ is the transmitted light intensity and $I_{\max }$ and $I_{\min }$ are the maximum and minimum intensity of the LC sample cell, respectively.

The $V-T$ curve as shown in figures 4 and 5 follows oscillatory nature and the number of maxima in the $V-T$ curve is approximately equal to $\Delta n \cdot d / \lambda$, which can be verified by substituting the value of $\Delta n, d$ and $\lambda$. Additionally, in a lowvoltage range $(0-1.6 \mathrm{~V})$ the output intensity through LC cells is found almost constant and a marked decrease in intensity was noticed beyond $1.6 \mathrm{~V}$. The threshold voltage $\left(V_{\mathrm{th}}\right)$ as observed from $V-T$ plots was 1.6 and $1.5 \mathrm{~V}$, respectively, for E7 and E7-ZnO sample cells. This minute decrease in $V_{\text {th }}$ after dispersion of NPs indicates changes in the retardation and birefringence of E7 at a higher voltage [9]. Further, field screening effects of impurity ions and a larger size of NPs as compared to LC molecules result in a larger dipole moment of NPs than LCs. A larger dipole moment of NPs gives rise to a high electric torque which affects the anchoring condition, which might cause a decrease in $V_{\text {th }}$ [9].

As we increase the voltage, the light transmission starts to vary and shows the maximum and minimum peaks which correspond to an odd or an even integer of equation (1). Above $5 \mathrm{~V}$, it is observed that the transmittance begins to decrease and tends to a minimum value in both E7 and E7-ZnO. Initially, in a low-voltage range $(<2 \mathrm{~V})$ the LC molecules have their orientation parallel to the glass substrates and show a higher transmittance. However, beyond $2 \mathrm{~V}$, the orientation of molecules starts to change from planar to homeotropic and finally shows a dark state or very lowtransmittance.

Figure 6 shows the temperature dependence on $\Delta n$ for E7 and $\mathrm{E} 7-\mathrm{ZnO}$ samples at $5 \mathrm{~V}$ (frequency $1 \mathrm{kHz}$ ). Above $30^{\circ} \mathrm{C}$, there is a decrease in the value of $\Delta n$ for both E7 and E7$\mathrm{ZnO}$ samples and approaches a minimum value near isotropic phase transition temperature. Here, the transmission method is used to measure $\Delta n$ for $\mathrm{E} 7$ and $\mathrm{E} 7-\mathrm{ZnO}$ samples [38,39]. Using equations ( 1 and 2), the calculated values of $\Delta n$ at $30^{\circ} \mathrm{C}$ are found to be 0.22 and 0.23 for $\mathrm{E} 7$ and $\mathrm{E} 7-\mathrm{ZnO}$, respectively, which is in good agreement with earlier work $[39,40]$. An increase in $\Delta n$ after dispersion of NPs can be attributed to the formation of large dipole moments, which produces a large electric field between NPs. Further, a decrease in birefringence with temperature as shown in figure 6 can be due to a change in molecular re-orientation and optical anisotropy of LCs. This shows that the system is moving to a less ordered 


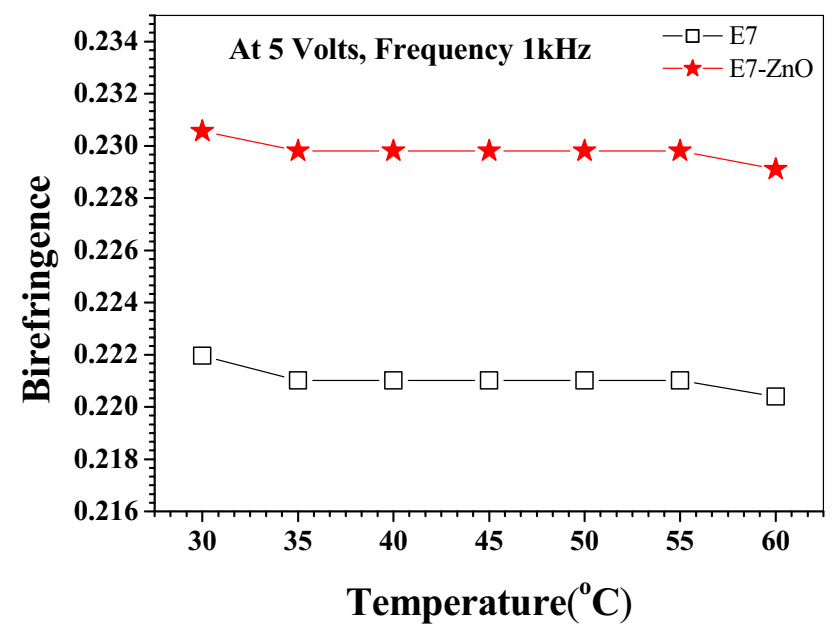

Figure 6. Variation of birefringence $(\Delta n)$ with temperature for $\mathrm{E} 7$ and $\mathrm{E} 7-\mathrm{ZnO}$ samples.

state with an increase in the kinetic energy of the system and a decrease in optical anisotropy [31,40].

Additionally, the contrast ratio (CR), an important measure of the performance for any display device and defined as $I_{\max } / I_{\min }$, is calculated from the maximum and minimum transmission data $[41,42]$. Our results show that the CR also improves after dispersion of ZnO NPs (figure 7). The change in the CR for the NP-dispersed sample can be attributed to molecular alignment ability of NPs, which alters the alignment of LC molecules and improve the optical properties of LCs [43]. Variation of the CR with temperature for E7 and E7$\mathrm{ZnO}$ samples is shown in figure 7 and a decrease in the $\mathrm{CR}$ was observed with an increase in temperature from the nematic to isotropic phase, which is due to fact that an increase in temperature increases disorder of LC molecules and affects the CR [44].

\subsection{Dielectric measurements}

Dielectric permittivity $\left(\varepsilon^{\prime}\right)$ and dielectric loss $\left(\varepsilon^{\prime \prime}\right)$ can be calculated by:

$$
\varepsilon^{\prime}=\frac{C_{\mathrm{LC}}-C_{\mathrm{A}}}{C_{\text {effective }}}+1
$$

and

$$
\varepsilon^{\prime \prime}=D \times \varepsilon^{\prime} .
$$

Here, $C_{\mathrm{LC}}$ is the capacitance of the cell filled with $\mathrm{LCs}$ and $C_{\mathrm{A}}$ is the capacitance of the empty cell (air) and

$$
C_{\text {effective }}=\frac{C_{\mathrm{B}}-C_{\mathrm{A}}}{\left(\varepsilon_{\mathrm{B}}^{\prime}-1\right)}
$$

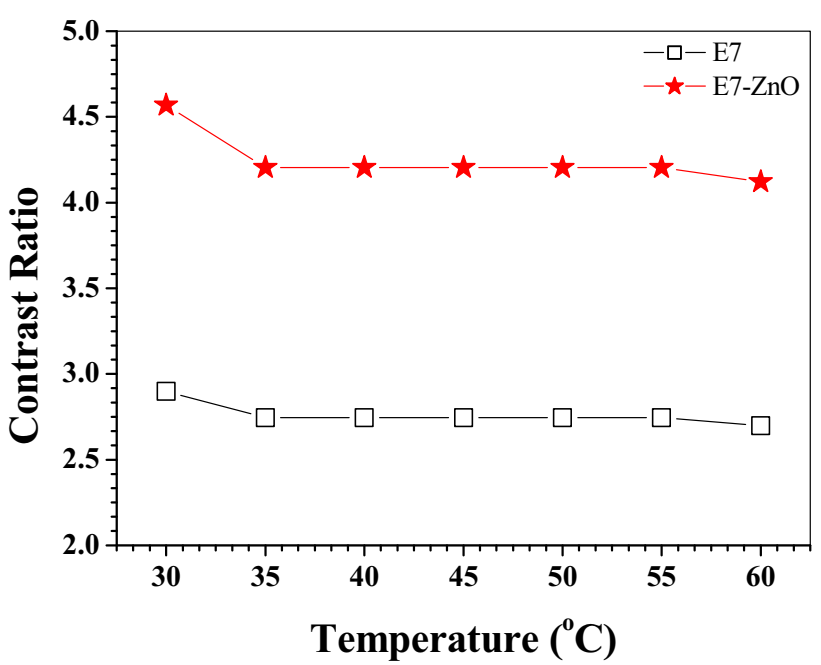

Figure 7. Variation of the $\mathrm{CR}$ with temperature for $\mathrm{E} 7$ and $\mathrm{E} 7-\mathrm{ZnO}$ samples.

Here, $C_{\mathrm{B}}$ is the capacitance of the cell filled with benzene, $\varepsilon_{\mathrm{B}}^{\prime}$ is the dielectric permittivity of benzene solution $\left(\varepsilon_{\mathrm{B}}^{\prime}=2.245\right)$ and $D$ is the dissipation factor. Here, it is worth mentioning that the complete removal of benzene was assured by heating the sample cell above the boiling point of benzene $\left(80^{\circ} \mathrm{C}\right)$. The cell was further examined through the POM to make it completely trace free of benzene.

The dielectric relaxation spectrum can be described with the help of the generalized Cole-Cole equation and is given by $[14,35,43-47]$ :

$$
\begin{aligned}
\varepsilon^{*}= & \varepsilon^{\prime}(\infty)+\sum \frac{\delta \varepsilon}{1+(j \omega \tau)^{(1-\alpha)}}+\frac{A}{\omega^{n}}-j \frac{\sigma_{\text {ion }}}{\varepsilon_{0} \omega^{k}} \\
& -j B \omega^{m} .
\end{aligned}
$$

On separating the real and imaginary part, equation (7) can be written as:

$$
\begin{aligned}
\varepsilon^{\prime}= & \varepsilon^{\prime}(\infty)+\sum \frac{\delta \varepsilon\left[1+(\omega \tau)^{(1-\alpha)} \sin (\alpha \pi / 2)\right]}{1+(\omega \tau)^{2(1-\alpha)}+2(\omega \tau)^{1-\alpha} \sin (\alpha \pi / 2)} \\
& +\frac{A}{\omega^{n}}, \\
\varepsilon^{\prime \prime}= & \sum \frac{\delta \varepsilon(\omega \tau)^{(1-\alpha)} \cos (\alpha \pi / 2)}{1+(\omega \tau)^{2(1-\alpha)}+(\omega \tau)^{1-\alpha} \sin (\alpha \pi / 2)} \\
& +\frac{\sigma_{\text {ion }}}{\varepsilon_{0} \omega^{k}}+B \omega^{m},
\end{aligned}
$$

where $\delta \varepsilon$ represents the dielectric strength of relaxation, $\varepsilon^{\prime}(\infty)$ represents the high-frequency limit of dielectric permittivity, $\alpha(0<\alpha<1)$ is the distribution parameter, $\varepsilon_{0}$ is the permittivity of free space, $\omega$ is the angular frequency and $\tau$ is the relaxation time.

The low- and high-frequency deviation in dielectric data require correction for low- and high-frequency values. Third 

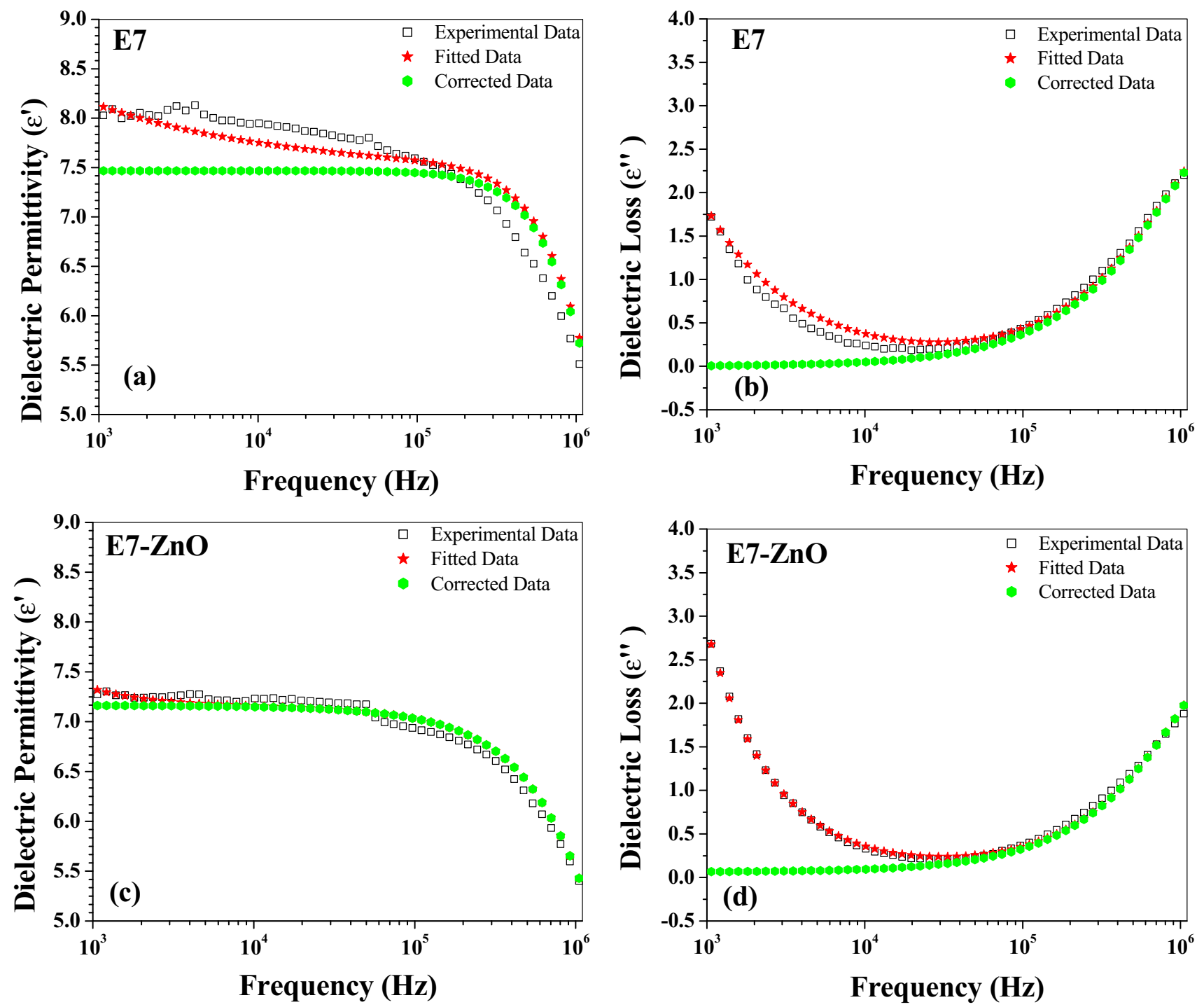

Figure 8. Frequency dependence of dielectric permittivity $\left(\varepsilon^{\prime}\right)$ and dielectric loss $\left(\varepsilon^{\prime \prime}\right)$ for (a, b) E7 and (c, d) E7-ZnO respectively, at $30^{\circ} \mathrm{C}$. Plots reflect the effect of fitting and correction on experimental data.

and fourth terms in equation (7) represent the contribution due to electrode polarization capacitance and ionic conductivity at low-frequencies. The observed value of dielectric absorption contains a contribution above $100 \mathrm{kHz}$ which is due to the finite resistance of electrodes and lead inductance. Therefore, an additional imaginary term $B \omega^{m}$ is included in equation (7) in order to partially account for the high-frequency effect. Here, $A, B, m, n$ and $k$ are the fitting parameters $[48,49]$.

The frequency dependence of $\varepsilon^{\prime}$ and $\varepsilon^{\prime \prime}$ for $\mathrm{E} 7$ and $\mathrm{E} 7-\mathrm{ZnO}$ samples in the nematic phase $\left(30^{\circ} \mathrm{C}\right)$ is shown in figure $8 \mathrm{a}-$ d. The obtained dielectric data were separately fitted with equations (8 and 9) using origin 6.1 software. In order to find best fit values, the fitting process with variation in fitting parameters was repeated until, chi-square $\left(\chi^{2}\right) \rightarrow 0$ and correlation coefficients $\left(R^{2}\right) \rightarrow 1$ are reached. In order to obtain correct values (free from low- and high-frequency effects), low- and high-frequency correction terms were acquired from the fitting process and then subtracted from the measured dielectric data. The purpose of fitting was to eliminate low(ionic conductance) and high-frequency correction (electrode and lead conductance) factors from the experimental values of permittivity and dielectric loss.

From figure 8a and c, we can infer that the dispersion of $\mathrm{ZnO}$ NPs leads to an insignificant decrease in the permittivity values from 7.4 to 7.0. Generally, it is observed that dispersion of NPs in LCs increases the dielectric permittivity due to an increase in the number of ions after dispersion, however in the present study, a minimal decrease or very less effect on permittivity of E7 was observed. This abnormal behaviour of permittivity in the E7-ZnO sample can be explained on the basis of the dopant size. In this experiment an average size of $\mathrm{ZnO}$ NPs is much larger $(\sim 50 \mathrm{~nm})$ as compared with 


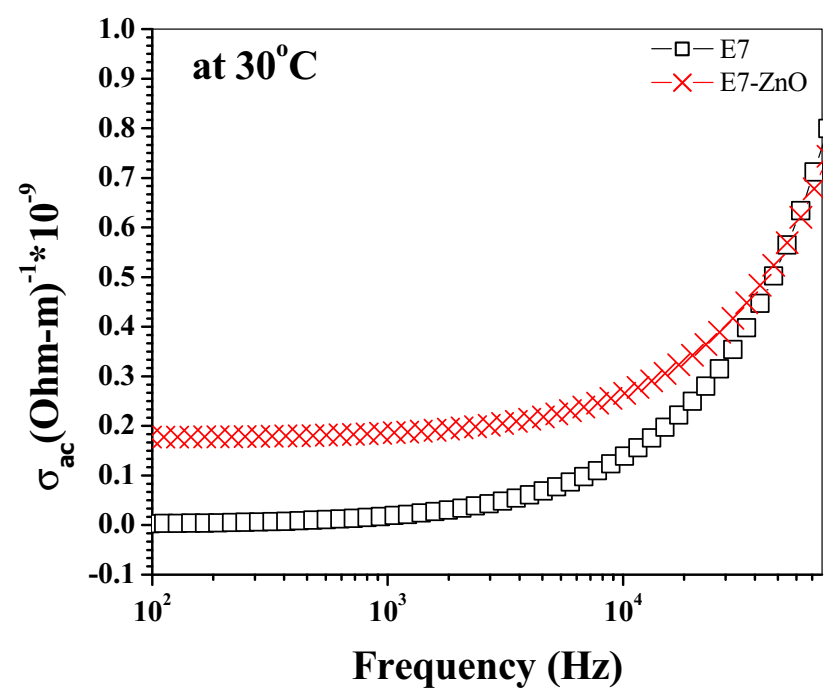

Figure 9. Frequency dependence of conductivity for E7 and E7$\mathrm{ZnO}$ samples in the nematic phase at $30^{\circ} \mathrm{C}$. Constant portion represents $\mathrm{dc}$ or ionic part whereas the increasing term above $\sim 0.2$ is dipolar or ac.

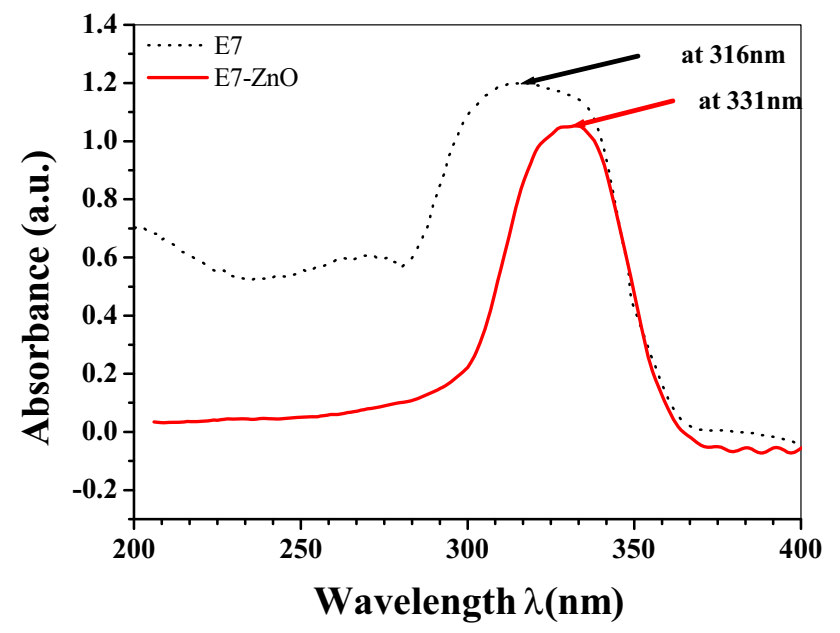

Figure 10. Absorbance characteristics of samples E7 and E7-ZnO.

LC molecules. This size difference results in a decrease in relative permittivity of E7. The decrease in the relative permittivity can be attributed to electronic charge transfer taking place through $\mathrm{ZnO}$ NPs. Due to a large size of ZnO NPs, the minimal distance between neighbouring NPs decreases to a sufficiently low-value. This provides an easier way of charge transfer through ZnO NPs [14]. Further, an analysis of absorption spectra shows that no relaxation mode was observed in the frequency range up to $1 \mathrm{MHz}$ in $\mathrm{E} 7$ and $\mathrm{E} 7-\mathrm{ZnO}$ samples. As shown in figure $8 \mathrm{~b}$ and $\mathrm{d}$, the dielectric value is very low and does not vary with the $\mathrm{ZnO}$ dopant.
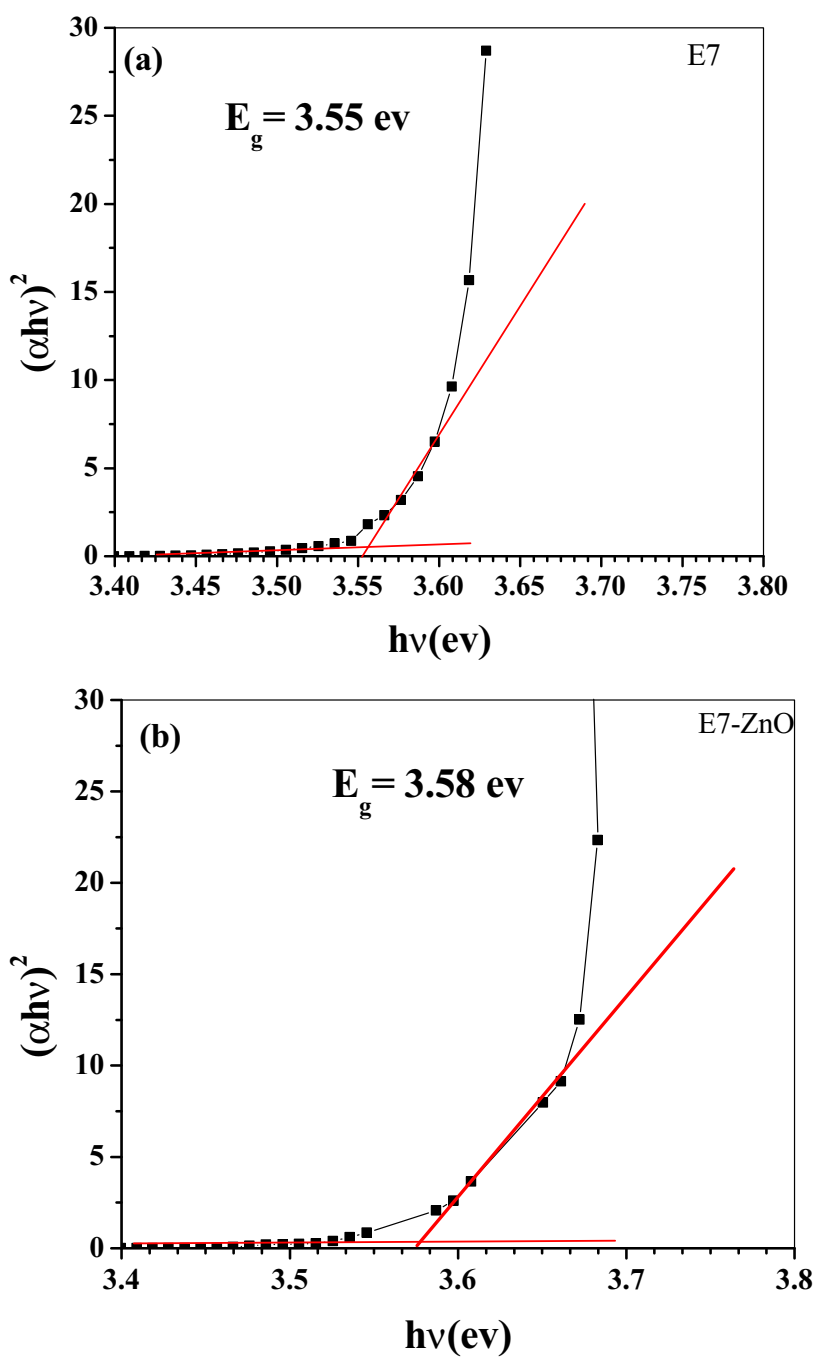

Figure 11. Estimation of the energy band gap of samples (a) E7 and (b) E7-ZnO.

\subsection{Conductivity and energy band gap}

Frequency dependence on conductivity has been investigated for E7 and E7-ZnO samples. The alternating current (ac) conductivity was examined using the equation [35]:

$$
\sigma(\omega)=\omega \varepsilon_{0} \varepsilon^{\prime \prime}, \quad \text { where } \omega=2 \pi f \text {. }
$$

Thus,

$$
\sigma(\omega)=2 \pi f \varepsilon_{0} \varepsilon^{\prime \prime},
$$

where $f$ is the frequency. Figure 9 shows the frequency dependence of conductivity for E7 and E7-ZnO samples in the nematic phase at $30^{\circ} \mathrm{C}$. The constant portion of the frequency as shown in the graph represents direct current (dc) or ionic part, whereas the increasing term above $\sim 0.2$ is dipolar or ac conductivity $[35,50]$ and the contribution of dc to dielectric loss in this region is negligible. The dispersion of $\mathrm{ZnO}$ 
Table 1. Electro-optic and dielectric parameters observed after dispersion of ZnO NPs in the nematic LC.

\begin{tabular}{lccccccc}
\hline Sample & $T_{\mathrm{N}-\mathrm{I}}\left({ }^{\circ} \mathrm{C}\right)$ & $\Delta n$ & $V_{\mathrm{th}}(\mathrm{V})$ & $\varepsilon^{\prime}$ & Absorbance peak wavelength $(\mathrm{nm})$ & $E_{\mathrm{g}}(\mathrm{eV})$ & $\sigma_{\mathrm{ac}}(\Omega \mathrm{m})^{-1} \times 10^{-9}$ \\
\hline E7 & 60.5 & 0.22 & 1.6 & 7.4 & 316 & 3.55 & 0.011 \\
E7-ZnO & 61.5 & 0.23 & 1.5 & 7.0 & 331 & 3.58 & 0.185 \\
\hline
\end{tabular}

NPs in E7 increases the ac conductivity of E7. An increase in conductivity after dispersion of ZnO NPs in E7 can be ascribed to a change in ion-concentration. Additionally, dispersion of $\mathrm{ZnO}$ NPs increases the charge carrier mobility and the concentration of free ions by ion transfer along the surface of interconnected particles [27,35]. Further, the absorbance characteristics of E7 and E7-ZnO samples are shown in figure 10. It is clearly seen that with dispersion of ZnO NPs, the absorption peak shifts to a higher wavelength side which was observed at 316 and $331 \mathrm{~nm}$ for E7 and E7-ZnO samples, respectively. Additionally, the energy band gap $\left(E_{\mathrm{g}}\right)$ has been calculated using the following Tauc relationship:

$$
\begin{aligned}
& a h v=A\left(h v-E_{\mathrm{g}}\right)^{n}, \\
& \alpha=-\frac{\ln T}{d},
\end{aligned}
$$

where $T$ is the transmission, $\alpha$ is the absorption coefficient, $d$ is the film thickness, $h v$ is the incident photon energy, $A$ is the constant, $E_{\mathrm{g}}$ is the energy band gap corresponds to the particular transition in the material and $n$ is an index having values $1 / 2,3 / 2,2$ and 3 depending on the nature of transition. Here, $n$ is $1 / 2$ for direct-allowed transition, $3 / 2$ for indirect-forbidden transition, 2 for indirect-allowed transition and 3 for direct-forbidden transition. Here, $n=1 / 2$ is selected for direct allowed transition $[35,51,52]$. The value of $E_{\mathrm{g}}$ was calculated from the graph between $(a h v)^{1 / n}$ and $h v$ as shown in figure 11. By extrapolation of the linear region at $\alpha=0$, the intercept of the linear fit on the $x$-axis gives $E_{\mathrm{g}}$. From figure $11 \mathrm{a}$ and $\mathrm{b}, E_{\mathrm{g}}$ is found to be 3.55 and $3.58 \mathrm{eV}$ for $\mathrm{E} 7$ and $\mathrm{E} 7-\mathrm{ZnO}$ samples, respectively. The increased value of $E_{\mathrm{g}}$ is expected due to an increase in charge carrier mobility with the concentration of $\mathrm{ZnO}$ NP free ions. Table 1 summarizes the results of electro-optic and dielectric variations observed after dispersion of $\mathrm{ZnO}$ NPs in nematic LCs.

\section{Conclusions}

Present work demonstrates the effect of $\mathrm{ZnO}$ NP dispersion on textural, electro-optic and dielectric properties of nematic LCs. Dark and bright states appeared as expected from LC orientational behaviour with an applied voltage. A slight increase $\left(\sim 1^{\circ} \mathrm{C}\right)$ in isotropic-nematic phase transition temperature of LCs was observed due to dispersion of NPs. NP dispersion in
LCs changes the anchoring condition and results in a decrease in the threshold voltage. The results also confirm a negligible decrease in the permittivity from 7.4 to 7.0 with NP dispersion, however relaxation frequency was not observed up to $1 \mathrm{MHz}$. Further, an energy band gap and ac conductivity were found to be increased for the LC-NP composite and is expected due to increased charge carrier mobility of $\mathrm{ZnO}$ NP free ions. The improved electro-optical characteristics i.e., increase in birefringence and CR with reduced threshold voltage after dispersion of NPs lead to less power consumption and better optical performance suitable for LC-based devices.

\section{Acknowledgements}

The authors sincerely thank the Director, NIT Jalandhar for continuous encouragement and support. The authors also thank DST, SERB and CSIR India for financial support under the Project Numbers EMR/2016/003560 and CSIR/031451/18/EMR-II, respectively.

\section{References}

[1] Nayek P, Karan S, Kundu S, Lee S H, Gupta S D, Roy S K et al 2012 J. Phys. D: Appl. Phys. 45235303

[2] de Gennes P G 1974 The physics of liquid crystals (Oxford: Clarendon Press)

[3] Robbie K, Broer D J and Brett M J 1999 Nature 399764

[4] Grollau S, Abbott N L and de Pablo J J 2003 Phys. Rev. E 67 051703

[5] Fukuda J, Yoneya M and Yokoyama H 2002 Phys. Rev. E 65 041709

[6] Loudet C J, Barois P and Poulin P 2000 Nature 407611

[7] Andrienko D, Allen M P, Skacej G and Zumer S 2002 Phys. Rev. E 65041702

[8] Woltmann S J, Jay G D and Crawford G P 2007 Nat. Mater. 6 929

[9] Eskalen H, Ozgan S, Alver U and Kerli S 2015 Acta Phys. Pol. A 127756

[10] Vardanyan K K, Palazzo E D and Walton R D 2011 Liq. Cryst. 38709

[11] Dolgov L, Yaroshchuk O and Lebovka M 2008 Mol. Cryst. Liq. Cryst. 496212

[12] Tripathi P K, Misra A K, Pandey K K and Manohar R 2013 Chem. Rapid Commun. 120 
[13] Chen W T, Chen P S and Chao C Y 2009 Jpn. J. Appl. Phys. 48015006

[14] Manohar R, Yadav S P, Srivastava A K and Misra A K 2009 Jpn. J. Appl. Phys. 48101501

[15] Reznikov Y, Buchnev O, Tereshchenko O, Reshetnyak V, Glushchenko A and West J 2003 Appl. Phys. Lett. 821917

[16] Li F, Buchnev O, Cheon C I, Glushchenko A, Reshetnyak V, Reznikov Y et al 2006 Phys. Rev. Lett. 97147801

[17] Lee W, Wang C Y and Shih Y C 2004 Appl. Phys. Lett. 85513

[18] Lee C W and Shih W P 2010 Mater. Lett. 64466

[19] Maleki A, Ara M H M and Saboohi F 2016 Phase Transit. 90 371

[20] Brochard F and de Gennes P G 1970 J. Phys. France 31691

[21] Rahman M and Lee W 2009 J. Phys. D: Appl. Phys. 42063001

[22] Misra A K, Srivastava A K, Shukla J P and Manohar R 2008 Phys. Scr. 78065602

[23] Allagulov A I, Pikin S A and Chigrinov V G 1989 Liq. Cryst. 51099

[24] Zennyoji M, Yokoyama J, Takanishi Y, Ishikawa K, Takezoe H and Itoh K 1998 Jpn. J. Appl. Phys. 376071

[25] Lagerwall S T 1999 Ferroelectric and antiferroelectric liquid crystals (Weinhelm: Wiley-VCH) p 200

[26] Wu P C, Yang S Y and Lee W 2016 J. Mol. Liq. 218150

[27] Tomylko S, Yaroshchuk O, Kovalchuk O, Maschke U and Yamaguchi R 2012 Ukr. J. Phys. 5239

[28] Singh U B, Dhar R, Dabrowski R and Pandey M B 2014 Liq. Cryst. 41953

[29] Singh U B, Dhar R, Dabrowski R and Pandey M B 2013 Liq. Cryst. $\mathbf{4 0} 774$

[30] Kurochkin O, Mavrona E, Apostolopoulos V, Blach J-F, Henninot J-F, Kaczmarek Met al 2015 Appl. Phys. Lett. 10604311

[31] Roy A, Pathak G, Herman J, Inamdar S R, Srivastava A and Manohar R 2018 Appl. Phys. A 124273

[32] Malik A, Choudhary A, Silotia P and Biradar A M $2011 J$. Appl. Phys. 110064111
[33] Al-Zangana S, Turner M and Dierking I 2017 J. Appl. Phys. 121085105

[34] Kumar P, Debnath S, Rao N V S and Sinha A 2018 J. Phys. Condens. Matter 30095101

[35] Khushboo, Sharma P, Malik P and Raina K K 2017 Liq. Cryst. 441717

[36] Prasad A and Das M K 2010 J. Phys. Condens. Matter 22 195106

[37] West J L, Zhang G and Glushchenko A 2005 Appl. Phys. Letts. 86031111

[38] Pande M, Tripathi P K, Gupta S K, Manohar R and Singh S 2015 Liq. Cryst. 421465

[39] Basu R and Shalov S A 2017 Phys. Rev. E. 96012702

[40] Mavrona E, Chodorow U, Barnes M E, Parka J, Palka N, Saitzek S et al 2015 AIP Adv. 5077143

[41] Montgomery G P Jr and Vaz N A 1987 Appl. Opt. 26738

[42] Raina K K, Kumar P and Malik P 2006 Bull. Mater. Sci. 29 599

[43] Singh D P, Pandey S, Gupta S K, Manohar R, Daoudi A, Sahraoui A H et al 2016 J. Lumin. 173250

[44] Porov P, Chandel V S and Manohar R 2016 Trans. Electr. Electron. Mater. 1769

[45] Koneracka M, Kellnerova V, Kopcansky P and Kuczynski T 1995 J. Magn. Magn. Mater. 1401455

[46] Cole K S and Cole R H 1941 J. Chem. Phys. 9341

[47] Gouda F, Skarp K and Lagerwall S T 1991 Ferroelectric 113 165

[48] Dhar R 2004 Ind. J. Pure Appl. Phys. 4256

[49] Chaudhary A, Malik P, Mehra R and Raina K K 2012 Phase Transit. 85244

[50] Srivastava S L and Dhar R 1996 Radiat. Phys. Chem. 47287

[51] Khushboo, Sharma P, Malik P and Raina K K 2016 Liq. Cryst. 431671

[52] Hassanien A S and Akl Alaa A 2016 Superlattices Microstruct. 89153 